\title{
Terrestrial atmosphere, water and astrobiology
}

\author{
A. Brack ${ }^{1, a}$ and M. Coradini ${ }^{2}$ \\ ${ }^{1}$ Centre de Biophysique Moléculaire, CNRS, Rue Charles Sadron, 45071 Orléans Cedex 2, France \\ ${ }^{2}$ ESA, 8-10 rue Mario Nikis, 75015 Paris, France
}

\begin{abstract}
Primitive life, defined as a chemical system capable to transfer its molecular information via self-replication and also capable to evolve, originated about 4 billion years ago from the processing of organic molecules by liquid water. Terrestrial atmosphere played a key role in the process by allowing the permanent presence of liquid water and by participating in the production of carbon-based molecules. Water molecules exhibit specific properties mainly due to a dense network of hydrogen bonds. The carbon-based molecules were either home made in the atmosphere and/or in submarine hydrothermal systems or delivered by meteorites and micrometeorites. The search for possible places beyond the earth where the trilogy atmosphere/water/life could exist is the main objective of astrobiology. Within the Solar System, exploration missions are dedicated to Mars, Europa, Titan and the icy bodies. The discovery of several hundreds of extrasolar planets opens the quest to the whole Milky Way.
\end{abstract}

\section{Introduction}

Defining life is a difficult task and the intriguing and long lasting question "What is life" has not yet received a commonly accepted answer, even for what could define a minimal life, the simplest possible form of life. Perhaps the most general working definition is that adopted by the NASA Exobiology Programme: "Life is a self-sustained chemical system capable of undergoing Darwinian evolution". Implicit in this definition is the fact that the system uses external matter and energy provided by the environment. In other words, primitive life can be defined, a minima, as an open chemical system capable of self-reproduction, i.e. making more of itself by itself, and also capable to evolve. The concept of evolution implies that the chemical system normally transferred its information fairly faithfully but made a few random errors, thus potentially leading to a higher complexity/efficiency and possibly to a better adaptation to changes in the environmental constraints.

Schematically, the premises of primitive life can be compared to parts of a "chemical automaton". By chance, some parts, self-assembled to generate automata capable of assembling other parts to form an identical chemical system. Sometimes, a minor error in the building generated a more efficient automaton which became the dominant species.

It is generally believed that the primitive automaton emerged in liquid water and that the parts were organic molecules, i.e. molecules that contained carbon and hydrogen atoms associated with oxygen, nitrogen and sulphur atoms, like present life. As parts of an open system, the constituents of a living system must be able to diffuse at a reasonable rate. Solid state life is generally discarded, the constituents being unable to migrate and to be easily exchanged. A gaseous phase would allow fast diffusion of the parts but the limited inventory of stable volatile organic molecules would constitute

\footnotetext{
a e-mail: brack@cnrs-orleans.fr
}

This is an Open Access article distributed under the terms of the Creative Commons Attribution-Noncommercial License 3.0, which permits unrestricted use, distribution, and reproduction in any noncommercial medium, provided the original work is properly cited. 
a severe restriction. A liquid phase offers the best environment for the diffusion and the exchange of dissolved organic molecules.

Terrestrial atmosphere played a key role in the emergence of life in allowing the permanent presence of oceanic water and also in participating in the production of organic molecules.

Is this trilogy atmosphere/water/life universal? Are there other places, within the Solar System and beyond, where such a trilogy could exist? Answering these questions is the main objective of astrobiology, also sometimes called exobiology.

\section{Atmosphere and water}

\subsection{Oceanic water}

Liquid water can persist only under a pressure higher than 6 mbars. Liquid water is therefore a rather fleeting substance. However, it was probably permanently present at the surface of the Earth thanks to the size of the planet and to its distance to the Sun [1]. If the planet happened to be much smaller, like Mercury or the Moon, it would not have been able to retain any atmosphere and, therefore, any liquid water. If the planet were too close to the star, the mean temperature would have raised due to starlight intensity. Any seawater present would evaporate delivering large amounts of water vapour to the atmosphere thus contributing to the greenhouse effect. Such a positive feedback loop could lead to a runaway greenhouse: all of the surface water would be transferred to the upper atmosphere where photo-dissociation by ultraviolet light would break the molecules into hydrogen, which would escape into space, and oxygen which would recombine into the crust.

The permanent presence of liquid water requires also a temperature above $0{ }^{\circ} \mathrm{C}$, although the freezing point of water can be depressed in the presence of salts (brines). For instance, the 5.5\% (by weight) salinity of the Dead Sea depresses the freezing point of sea water by about $3{ }^{\circ} \mathrm{C}$. Large freezing point depressions are observed for $15 \% \mathrm{LiCl}\left(23.4^{\circ} \mathrm{C}\right)$ and for $22 \% \mathrm{NaCl}\left(19.2^{\circ} \mathrm{C}\right)$. The positive temperature was mainly due to a constant greenhouse atmosphere. Although the true composition of primitive Earth's atmosphere remains difficult to define, the dominant view in recent years is that the primitive atmosphere consisted mainly of $\mathrm{CO}_{2}, \mathrm{~N}_{2}$, and $\mathrm{H}_{2} \mathrm{O}$, along with small amounts of $\mathrm{CO}$ and $\mathrm{H}_{2}[2,3]$. An efficient greenhouse effect induced by a high $\mathrm{CO}_{2}$ partial pressure was the most likely mechanism capable of compensating for the faint young Sun, the luminosity of which was about $30 \%$ lower than today. However, water risked to provoke its own disappearance. The atmospheric greenhouse gas $\mathrm{CO}_{2}$ normally dissolves in the oceans and is finally trapped as insoluble carbonates by silicate rockweathering. This negative feedback is expected to lower the surface pressure and the temperature to an extent that water would be largely frozen. On Earth, active plate tectonics and volcanism recycled the carbon dioxide by breaking down subducted carbonates. If the temperature were to drop below freezing, silicate weathering would slow down and volcanic $\mathrm{CO}_{2}$ would accumulate in the atmosphere. Prior to the emergence of the continents, the $\mathrm{CO}_{2}$ partial pressure could have been as high as 10 bars because of limited silicate weathering and limited storage in continental carbonate minerals [4]. Under these conditions, the early Earth could have been as hot as $85^{\circ} \mathrm{C}$. Analysis of samarium/neodymium ratios in the ancient zircons indicate that the continents grew rapidly, so a dense $\mathrm{CO}_{2}$ atmosphere may have been restricted to the first few hundred million years of the Earth's history.

The source of the primitive terrestrial atmosphere can be found in a combination of the volatiles $\mathrm{CO}_{2}, \mathrm{~N}_{2}, \mathrm{H}_{2} \mathrm{O}$, trapped in the rocks that made the bulk of the planet's mass and a late accreting veneer of extraterrestrial material. The atmosphere produced by the planet's early accretion, which was subsequently blown off by the giant impact between the Earth and a Mars-sized planetary embryo that generated the Moon 50 to 70 million years after the formation of the Earth [5] and caused the early atmosphere to undergo massive hydrodynamic loss, may have been replaced by a late volatilerich veneer via meteorites $[6,7]$ and micrometeorites $[8]$.

\subsection{The virtues of liquid water}

According to its molecular weight, water should be a gas under standard terrestrial conditions by comparison with $\mathrm{CO}_{2}, \mathrm{SO}_{2}, \mathrm{H}_{2} \mathrm{~S}$, etc. Its liquid state under standard conditions is due to its ability to form 
hydrogen bonds. This is not restricted to water molecules since alcohols exhibit a similar behaviour. However, the polymeric network of water molecules via $\mathrm{H}$-bonds is so tight that the boiling point of water is raised from $40^{\circ} \mathrm{C}$, a temperature inferred from the boiling point of the smallest alcohols, to $100^{\circ} \mathrm{C}$. The biopolymers, such as nucleic acids, proteins and membranes, contain CxHyO,N,S-groups and $\mathrm{CxHy}$-groups (hydrocarbon groups). Groups like $\mathrm{CxHyO}, \mathrm{N}, \mathrm{S}$, especially those bearing ionizable groups such as $-\mathrm{COOH}$ or $-\mathrm{NH}_{2}$, form hydrogen bonds with water molecules and display therefore an affinity for water. They are soluble in water and hydrophilic. The large dipole moment of water (1.85 debye) favours the dissociation of the ionizable groups while the high dielectric $(\varepsilon=80)$ prevents the ions to recombine, the attraction forces for ion re-association being proportional to $1 / \varepsilon$. This is also true for metallic ions which are associated with the biopolymers. CxHy-groups cannot form hydrogen bonds with water molecules and tend to escape water molecules as much as possible. They are insoluble in water and hydrophobic. These two groups co-exist in carbon chemistry and this co-existence drives the conformation (geometry) of the biopolymers in water, i.e. helices, sheets, micelles, vesicles or liposomes.

Water participates in the production of clays. Clay minerals are formed by water weathering of silicate minerals. As soon as liquid water was permanently present in the surface of the Earth, clay minerals accumulated and became suspended in the water reservoir. The importance of clay mineral in the origins of life was first suggested by Bernal [9]. The advantageous features of clays for Bernal were (i) their ordered arrangement, (ii) their large adsorption capacity, (iii) their shielding against sunlight, (iv) their ability to concentrate organic chemicals, and (v) their ability to serve as polymerisation templates. Since the seminal hypothesis of Bernal, many prebiotic scenarios involving clays have been written and many prebiotic experiments have used clays.

Water stabilises the biopolymer conformation by hydrophobic clustering. For example, when synthetic polypeptides based on a strict alternation of hydrophobic and hydrophilic amino acids are dissolved in water in the presence of salt, $\beta$-sheet bilayers are formed by clustering of the hydrophobic side-chains. When alcohol is added, the $\beta$-sheet structure is destroyed as a result of the relaxation of the water constrain on the hydrophobic clusters. Due to $\beta$-structure formation, strictly alternating hydrophobic-hydrophilic sequences are thermostable and resistant to chemical degradation [10].

Water is also a powerful hydrolytic chemical reactant. Laboratory experiments have shown that water drives chemical pathways which would not occur in a non aqueous milieu.

Water is a good heat dissipator. Deep-sea hydrothermal systems may also represent likely environments for the synthesis of prebiotic organic molecules but hydrothermal vents are often disqualified as efficient reactors for the synthesis of bio-organic molecules because of the high temperature. However, the products that are synthesised in hot vents are rapidly quenched in the surrounding cold water thanks to the good heat conductivity of water. When fluid containing glycine repeatedly circulated through the hot $\left(225^{\circ} \mathrm{C}\right)$ and cold $\left(0^{\circ} \mathrm{C}\right)$ regions in a laboratory reactor that simulated a hydrothermal system, glycine peptides up to octaglycine were obtained [11].

\subsection{Alternatives to water}

Water played a major role in the appearance and evolution of life. However, other possible biogenic liquids must not be ruled out. Ammonia is liquid between $-78{ }^{\circ} \mathrm{C}$ and $-33^{\circ} \mathrm{C}$ under one bar and can form hydrogen bonds to dissolve organic molecules. It is present as gas in interstellar dust clouds and as liquid droplets in the clouds of Jupiter but no large pool of liquid ammonia has been detected, so far. The same holds for formamide which is also liquid across a wide range of temperatures and pressures and a good solvent.

\section{Prebiotic carbon chemistry}

\subsection{The virtues of carbon chemistry}

Life is autocatalytic in essence and must be able to evolve. To evolve, i.e. improving the efficiency of self reproduction and increasing its diversity, the molecules bearing the hereditary memory must 
reach a certain level of complexity. This can be best achieved with a scaffolding of polyvalent atoms. In chemists' hands, tetravalent carbon atoms are very productive in this respect. It is hard to find any other element offering such a capability for building large molecules necessary for life. Siliconbased biochemistries in non-aqueous solvents are often suggested [12]. Situated just below carbon in the Mendeleev table of elements, it is also able to form four bonds at a time. The silicon atom is larger than the carbon one, so that less is formed in the cores of stars. There is therefore less silicon then carbon in the Universe. For example, 11 silicon containing molecules have been identified by radio astronomy versus 110 for carbon. Due to the mode of formation of the Solar System, silicon is much more abundant in the Earth's crust where it represents $27.7 \%$, versus $0.094 \%$ for carbon. Although silicon was always abundant on the primitive Earth, it has not been taken to start life. Being larger than carbon, the bonds made by silicon atoms with other atoms are generally weaker, thus producing more fragile polymers under prebiotic conditions. Very few double bonds and no triple bonds are known for silicon, a very efficient way to store chemical energy in carbon chemistry. Under extreme conditions of temperature and pressure, silicon can generate relatively stable polymers but they are insoluble in current solvents.

\subsection{Production of organics in oceanic water}

\subsubsection{Submarine hydrothermal systems}

The reducing conditions in submarine hydrothermal systems may have been an important source of biomolecules on the primitive Earth [13-15]. The reducing environment results from the flow of substances dissolved in sea water passing inorganic compounds present in very hot crustal material that reduce compounds in the sea water. These reduced compounds flow out of the hydrothermal system and the inorganic sulphides formed precipitate when they mix with the cold $\left(4^{\circ} \mathrm{C}\right)$ ocean water. For example, hydrocarbons containing 16 to 29 carbon atoms have been detected in the Rainbow ultramafic hydrothermal system, Mid-Atlantic Ridge [16]. Hydrothermal vents are often disqualified as efficient reactors for the synthesis of bioorganic molecules, because of the high temperature. However, the products that are synthesized in hot vents are rapidly quenched in the surrounding cold water, which may preserve those organics formed.

\subsubsection{The role of minerals}

Clay minerals are formed by water weathering of silicate minerals. As soon as liquid water was permanently present in the surface of the Earth, clay minerals accumulated and became suspended in the water reservoir. As already mentioned, the importance of clay mineral in the origins of life was first suggested by Bernal [9]. Since the seminal hypothesis of Bernal, many prebiotic experiments have been run with clays [17-19].

If the carbon source for life was carbon dioxide, the energy source required to reduce the carbon dioxide might have been provided by the oxidative formation of pyrite from iron sulphide and hydrogen sulphide. Pyrite has positive surface charges and bonds the products of carbon dioxide reduction, giving rise to a two-dimensional reaction system, a "surface metabolism" [20-22]. Laboratory work has provided some support for this promising hypothesis. An early laboratory simulation of hydrothermal synthetic reactions is the reduction of carbon dioxide to organic sulphides at $75^{\circ} \mathrm{C}$ in the presence of $\mathrm{FeS}$ and $\mathrm{H}_{2} \mathrm{~S}$. Methyl- and ethyl thiols were the principal thiols formed along with smaller amounts of others containing up to five carbon atoms. The $\mathrm{CO}_{2}$ was also converted to $\mathrm{CS}_{2}$ and $\mathrm{COS}$ [23]. The direct reduction of $\mathrm{CO}_{2}$ to acetic acid, acetaldehyde ethanol and smaller amounts of carbon compounds containing up to six carbon atoms, was observed to take place at $350^{\circ} \mathrm{C}$ and high pressure in the presence of magnetite $\left(\mathrm{FeO}: \mathrm{Fe}_{2} \mathrm{O}_{3}\right)$ and small amounts $(2 \%)$ of water [24].

There are also reports of the reaction of $\mathrm{CO}$ in simulated hydrothermal systems. When a mixture of $\mathrm{CO}$ and $\mathrm{CH}_{3} \mathrm{SH}$ was reacted with a combination of a NiS-FeS at $100^{\circ} \mathrm{C}$, acetic acid and its corresponding thioester were formed [25]. This system was extended to the formation of keto esters at higher temperatures and pressures where $\mathrm{CO}$ inserted into the thioester to form a keto thioester that 
in turn hydrolyzed to pyruvic acid [26]. More recently, $\alpha$-hydroxy and $\alpha$-amino acids were obtained under possible volcanic origin-of-life conditions by heating $\mathrm{CO}$ and $\mathrm{KCN}$ in the presence of nickel or nickel/iron catalysts [27]. However, the plausibility of the conditions used has been debated [28].

\subsubsection{Impact shock chemistry}

Furukawa and colleagues [29] investigated whether meteorite impacts could synthesize organic compounds. The group subjected a mixture of solid carbon, iron, nickel, water and nitrogen to high-velocity impacts in a propellant gun. This experiment simulated the chemistry experienced by ordinary chondrites when hitting the Earth's early oceans. They recovered several organic molecules after the impact, including complex molecules such as fatty acids and amines. Glycine, the simplest protein-building amino acid, was formed when the starting material contained ammonia, which is believed to have been formed in prior impacts on the early Earth. To avoid potential contamination by biological materials, the authors used solid carbon composed only of ${ }^{13} \mathrm{C}$. Subsequent analyses of the recovered organic products showed that they were all formed with the ${ }^{13} \mathrm{C}$, ruling out the presence of any naturally formed biological contaminants, which would have been enriched in ${ }^{12} \mathrm{C}$.

\subsection{Synthesis of organics in the atmosphere}

\subsubsection{The Miller synthesis}

Oparin [30] suggested that the small reduced organic molecules needed for primitive life were formed in a primitive atmosphere dominated by methane. The idea was tested in the laboratory by Miller [31] who exposed a mixture of methane, ammonia, hydrogen and water to spark discharge and silent electric discharge. In his initial experiment, he obtained three amino acids (glycine, alanine and $\beta$-alanine) via the intermediary formation of hydrogen cyanide and aldehydes. More generally, simple gaseous molecules, like $\mathrm{CH}_{4}, \mathrm{H}_{2}, \mathrm{NH}_{3}$, and $\mathrm{H}_{2} \mathrm{O}$, require a supply of energy (UV, heat, electric discharges, cosmic rays, shock waves) to react with each other. They generate compounds like formaldehyde and hydrogen cyanide which store chemical energy in their double and triple chemical bonds, respectively. The possible sources of atmospheric synthesis including electric effects, solar UV and impact shocks have been reviewed by Chang [32].

Miller's laboratory synthesis of amino acids occurs efficiently when a reducing gas mixture containing significant amounts of hydrogen is used. As already mentioned, the dominant view in recent years is that the primitive atmosphere consisted mainly of $\mathrm{CO}_{2}, \mathrm{~N}_{2}$, and $\mathrm{H}_{2} \mathrm{O}$, along with small amounts of $\mathrm{CO}$ and $\mathrm{H}_{2}$. Only small yields of amino acids are formed in such a mixture [33]. Recent studies show that the low yields previously reported appear to be the outcome of oxidation of the organic compounds during hydrolytic workup by nitrite and nitrate produced in the reactions. The yield of amino acids is greatly increased when oxidation inhibitors, such as ferrous iron, are added, suggesting that endogenous synthesis from neutral atmospheres may be more important than previously thought [34]. Additionally, twenty two amino acids and five amines were obtained when reanalyzing archived samples of experiment run by Miller and simulating production of organic molecules in volcanic gases by lightning. The volcanic apparatus experiment suggests that, even if the overall atmosphere was not reducing, localized prebiotic synthesis could have been effective in volcanic plumes [35].

The escape of hydrogen from early Earth's atmosphere has been re-evaluated [36]. It likely occurred at rates slower by two orders of magnitude than previously thought. The balance between slow hydrogen escape and volcanic outgassing could have maintained a hydrogen mixing ratio of more than $30 \%$, thus producing more amino acids than previously thought.

\subsubsection{Chemical reprocessing of the atmosphere by impacts}

The intense bombardment described below probably caused some chemical reprocessing of the Earth's primitive atmosphere by impact shock chemistry. Computer modelling of the impact shock chemistry 
shows that the nature of the atmosphere strongly influences the shock products [37]. A neutral $\mathrm{CO}_{2}$ rich atmosphere produces $\mathrm{CO}, \mathrm{O}_{2}, \mathrm{H}_{2}, \mathrm{NO}$ while a reducing $\mathrm{CO}$-rich atmosphere yields primarily $\mathrm{CO}_{2}, \mathrm{H}_{2}, \mathrm{CH}_{4}, \mathrm{HCN}, \mathrm{NH}_{3}$, and $\mathrm{H}_{2} \mathrm{CO}$. The last three compounds are particularly interesting for prebiotic chemistry since they can lead to amino acids via the Strecker synthesis. However, a CO-rich primitive atmosphere probably has no counterpart in prebiotic reality. In laboratory experiments, a gas mixture of methane, ammonia and water subjected to shock heating followed by a rapid thermal quenching yielded the amino acids glycine, alanine, valine and leucine [38]. Here again, the gas mixture used does not represent a realistic primitive atmosphere, which was dominated by $\mathrm{CO}_{2}$. Laboratory simulations of shocks were also run with a high-energy laser. $\mathrm{CH}_{4}$-containing mixtures generated hydrogen cyanide and acetylene but no organics could be obtained with $\mathrm{CO}_{2}$-rich mixtures [39].

\subsection{Atmosphere and the delivery of extraterrestrial organics}

The Earth experienced a large spectrum of impactors ranging from the huge Mars-sized impactor which created the Moon to cosmic dust less than $1 \mu \mathrm{m}$ in size. An indication of the number and timing of the impacts onto the early Earth can be obtained by comparison with the crater record of the Moon, which records impacts from the earliest history of the solar system [40]. Because of the larger size of the Earth and its greater gravitational pull, about 20 times as many impacts would have occurred on the early Earth as on the Moon. All these impactors crossed the atmosphere which acted as a shock absorber.

\subsubsection{Meteorites}

Carbonaceous chondrites delivered organic materials to the early Earth. They contain from $1.5 \%$ to $4 \%$ of carbon, for the most part as organic materials. One hundred kilograms of the Murchison meteorite, a CM2 type carbonaceous chondrite that fell in Australia in 1969, have been extensively analyzed [41,42]. Murchison organic materials are generally classified according to their solubility in water and organic solvents. Insoluble and soluble components represent respectively $70 \%$ and $30 \%$ of total carbon components. The insoluble organic material is referred as kerogen-like, a poorly identified insoluble macromolecular material of complex composition with average elemental abundances $\mathrm{C}_{100} \mathrm{H}_{46} \mathrm{~N}_{10} \mathrm{O}_{15} \mathrm{~S}_{4.5}$. NMR, IR and pyrolysis analyses suggest the presence of aromatic ring clusters bridged by aliphatic chains, with peripheral branching and functional groups. The insoluble organic material releases a variety of aromatic and heteroatomic hydrocarbons as well as a suite of alkyl dicarboxylic acids up to $\mathrm{C} 18$ chain length under conditions similar to those of hydrothermal vents [43].

The soluble organic compounds of the Murchison meteorite represent a diverse and abundant group of organics that vary from small water-soluble compounds such as amino acids and polyols up to 30 carbon-long hydrocarbons. This diversity has been analyzed in details for the amino acids. The total number of meteoritic amino acids is about one hundred. All the possible $\alpha$-amino alkylamino acids up to seven-carbon were identified as well as large abundances of $\mathrm{N}$-substituted, cyclic, $\beta-, \gamma-, \delta$-, and $\varepsilon$-amino acids. Eight protein-building amino acids (glycine, alanine, proline, leucine, isoleucine, valine, aspartic acid and glutamic acid) have been found. Nucleic acid bases, purines and pyrimidines, have also been found in the Murchison meteorite [44]. No ribose, the sugar moiety which links together the nucleic acid building blocks was detected in meteorites. Vesicle-forming fatty acids have been extracted from different carbonaceous meteorites [45].

A combination of high resolution analytical methods, composed of organic structural spectroscopy FTICR/MS, UPLC-QTOF-MS and NMR, applied to the organic fraction of Murchison extracted under mild conditions allowed to extend its indigenous chemical diversity to tens of thousands of different molecular compositions and likely millions of diverse structures [46].

Most of the amino acids detected in the carbonaceous chondrites are chiral but present as racemic, i.e. L- and D-enantiomers are present in equal proportions. However, Cronin and Pizzarello [47] found $\mathrm{L}$ enantiomer excesses in six $\alpha$-methyl- $\alpha$-amino alkanoic acids from the Murchison (2.8$9.2 \%)$ and Murray (1.0-6.0\%) carbonaceous chondrites. An enantiomeric excess up to $18 \%$, as been 
measured for isovaline, 2-methyl-2-aminobutyric acid. These amino acids (isovaline, 2-amino-2,3dimethylpentanoic, $\alpha$-methyl norvaline, $\alpha$-methyl valine and $\alpha$-methyl norleucine) are either unknown or rare in the terrestrial biosphere and cannot therefore be attributed to terrestrial contamination [41]. In addition, the indigeneity of D- and L-isovaline enantiomers is supported by carbon and hydrogen isotopic data $[48,49]$.

Several organic and inorganic phases of the carbonaceous chondrite were separated and subjected to the Soai reaction, i.e. the addition reaction of $\mathrm{i}-\mathrm{Pr}_{2} \mathrm{Zn}$ to pyrimidine-5-carbaldehyde. Asymmetric autocatalysis with amplification of chirality gave pyrimidyl alkanol with enantiomeric excesses of detectable level. The asymmetry resides in powders after extraction with water and solvents as well as in the insoluble organic material obtained after demineralization. Asymmetry is not found any longer in meteorite powders from which all organics had been removed [50].

The meteoritic enantiomeric excesses may help to understand the emergence of a homochiral (onehanded) life. Each amino acid, with the exception of glycine, exists under two enantiomeric forms, L and $\mathrm{D}$, but proteins use only $\mathrm{L}$ ones. Proteins adopt asymmetrical rigid geometries, $\alpha$-helices and $\beta$-sheets, which play a key role in the catalytic activity. Homochirality is now believed to be not just a consequence of life, but also a prerequisite for life, because stereoregular structures such as protein $\beta$-sheets, for example, do not form with mixtures of monomers of both handednesses. The use of one-handed biomonomers also sharpens the sequence information of the biopolymers. For a polymer made of $n$ units, the number of sequence combinations will be divided by $2 n$ when the system uses only homochiral monomers. Taking into account the fact that enzyme chains are generally made of hundreds of monomers, the tremendous gain in simplicity offered by the use of monomers restricted to one handedness is self evident. The excess of one-handed amino acids found in the Murchison meteorite may result from the processing of the organic mantles of interstellar grains by circularly polarized synchrotron radiation from a neutron star remnant of a supernova [51].

Strong infrared circular polarization, resulting from dust scattering in reflection nebulae in the Orion OMC-1 star-formation region, has been observed. Circular polarization at shorter wavelengths might have been important in inducing chiral asymmetry in interstellar organic molecules that could be subsequently delivered to the early Earth [52,53].

The effects of impact shock on amino acids and a peptide in artificial meteorites composed of saponite clay were investigated [54]. The samples were subjected to pressures ranging from 12 to $28.9 \mathrm{GPa}$, which simulated impact velocities of $2.4-5.8 \mathrm{~km} / \mathrm{s}$. Volatilization was determined by weight loss measurement, and the amino acid and peptide response was analyzed by gas chromatographymass spectrometry. At the highest shock pressures, amino acids with an alkyl side chain were more resistant than those with functional side chains. Impact shock may therefore act as a selective filter to the delivery of extraterrestrial amino acids via carbonaceous chondrites. The peptide cleaved into its two primary amino acids. Some chiral amino acids experienced partial racemization during the course of the experiment, suggesting that the enantiomeric excesses measured in carbonaceous chondrites are probably under evaluated.

\subsubsection{Micrometeorites}

Dust collections in the Greenland and Antarctica ice sheet $[8,55]$ show that the Earth captures interplanetary dust as micrometeorites at a rate of about 20000 tonnes per year. About $99 \%$ of this mass is carried by micrometeorites in the $50-500 \mu \mathrm{m}$ size range. This value is about 2,000 times higher than the most reliable estimate of the meteorite flux, i.e. about 10 tonnes per year. This amazing dominance of micrometeorites already suggests their possible role in delivering complex organics to the early Earth 4.2 to $3.9 \mathrm{Ga}$ ago when the micrometeorite flux was probably enhanced by several orders of magnitude. Antarctic micrometeorite flux measurements suggest that a huge mass $\left(\sim 5 \times 10^{24} \mathrm{~g}\right)$ of micrometeorites was accreted by the Earth during the first $\sim 300 \mathrm{Ma}$ of the post-lunar period.

At least, $\sim 20 \mathrm{wt} . \%$ of the micrometeorites survives unmelted upon atmospheric entry. As their kerogen fraction represents about $2.5 \mathrm{wt} \%$ of carbon, this amounts to a total mass of kerogen of $\sim 2.5 \times 10^{22} \mathrm{~g}$ on the early Earth surface equivalent to a $\sim 40 \mathrm{~m}$ thick global layer [56]. This delivery represents more carbon than that present in the biomass of the present day Earth $\left(10^{18} \mathrm{~g}\right)$. One amino 
acid, $\alpha$-amino isobutyric acid, has been identified in Antarctic micrometeorites [57,58]. These grains contain also a high proportion of metallic sulfides, oxides and clay minerals, a rich variety of inorganic catalysts which could have promoted the reactions of the carbonaceous material which lead to the origin of life. Analysis of the dust grains collected by the Cosmic Dust mission supports a cometary origin for the micrometeorites collected in Antarctica.

\subsubsection{The Rosetta mission}

Rosetta will be the first spacecraft to undertake the long-term study of a comet at close quarters. It is one of the most challenging missions ever undertaken and is due to rendezvous with Comet 67P Churyumov-Gerasimenko in 2014. The European Space Agency (ESA) mission comprises a large orbiter, designed to operate for a decade at large distances from the Sun, and a small lander called 'Philae'. Each of these carries a large complement of scientific experiments designed to complete the most detailed study of a comet ever attempted. After entering orbit around Comet 67P/ChuryumovGerasimenko in 2014, the spacecraft will release a small lander onto the icy nucleus, and then spend the next two years orbiting the comet as it heads towards the Sun. On the way to Comet ChuryumovGerasimenko, Rosetta receives gravity assists from Earth and Mars, and will fly past main belt asteroids.

The study of cometary material presents a major challenge due to the very characteristics that make it a unique repository of information about the formation of the Solar System, namely its high volatiles and organic-material contents. A fundamental question that the Rosetta mission has to address is, to what extent can the material accessible for analysis be considered representative of the bulk of the material constituting the comet, and of the early nebular condensates that constituted the cometesimal $4.57 \times 10^{9}$ years ago? This representativeness issue has to be addressed by first determining the global characteristics of the nucleus, namely its mass, density and state of rotation, which can provide us with clues as to the relationship between the comet's outer layers and the underlying material.

Comet 67P/Churyumov-Gerasimenko was discovered in 1969, when several astronomers from Kiev visited the Alma-Ata Astrophysical Institute to conduct a survey of comets. On 20 September, Klim Churyumov was examining photographs of comet 32P/Comas Solá taken by Svetlana Gerasimenko when he found a comet-like object near the edge of the plate. He assumed that the faint object was the expected periodic comet, but upon returning to Kiev, he studied the plates very carefully and eventually realised that a new comet had been found, less than two degrees from comet Comas Solá.

The comet has a particularly unusual history. Up to 1840 its perihelion distance was $4.0 \mathrm{AU}$ (four Sun-Earth distances or about 600 million $\mathrm{km}$ ) and the comet was completely unobservable from Earth. That year, a fairly close encounter with Jupiter caused the orbit to move inwards to a perihelion distance of $3.0 \mathrm{AU}$ ( 450 million $\mathrm{km}$ ). Over the next century, the perihelion gradually decreased further to 2.77 AU. Then, in 1959, a further Jupiter encounter reduced the perihelion to just 1.29 AU. It currently completes one orbit of the Sun every 6.57 years. It is unusually active for a short period object and has a coma (a diffuse cloud of dust and gas surrounding the solid nucleus) and often a tail at perihelion. During the 2002/2003 apparition, the tail was up to 10 arc minutes long as seen from Earth, with a bright central condensation in a faint extended coma. Even 7 months after perihelion the tail continued to be very well developed, although it subsequently faded rapidly. The comet typically reaches a magnitude around 12, although this is because the comet has outburst at perihelion at three of its last four returns in 1982/83, 1996/97 and 2002/03. Despite being a relatively active object, even at the peak of outburst the dust production rate is some 40 times lower than for 1P/Halley. Nevertheless, 67P/Churyumov-Gerasimenko is classed as a dusty comet. The peak dust production rate in 2002/03 was estimated at approximately $60 \mathrm{~kg}$ per second, although values as high as $220 \mathrm{~kg}$ per second were reported in 1982/83. The gas to dust emission ratio is approximately 2.

Sixty-one images of comet 67P/Churyumov-Gerasimenko were taken with the Wide Field Planetary Camera 2 on board the Hubble Space Telescope on 11-12 March 2003. The HST's sharp vision enabled astronomers to isolate the comet's nucleus from the coma. The images showed that the nucleus measures five by three kilometres and has an ellipsoidal (rugby ball) shape. It rotates once in approximately 12 hours. 
The above comet will be reached and explored by the Rosetta spacecraft. Rosetta is a large aluminium box with dimensions $2.8 \times 2.1 \times 2.0$ metres. The scientific instruments are mounted on the 'top' of the box (Payload Support Module) while the subsystems are on the 'base' (Bus Support Module). On one side of the orbiter is a 2.2-metre diameter communications dish, the steerable highgain antenna. The lander is attached to the opposite face. Two enormous solar panel 'wings' extend from the other sides. These wings, each 32 square metres in area, have a total span of about 32 metres tip to tip. Each of them comprises five panels, and both may be rotated through $+/-180$ degrees to catch the maximum amount of sunlight.

In the vicinity of Comet 67P/Churyumov-Gerasimenko, the scientific instruments almost always point towards the comet, while the antennae and solar arrays point towards the Sun and Earth (at large distances, they are more or less in the same direction).

A European consortium, under the leadership of the German Aerospace Research Institute (DLR), provides the 100-kilogram Rosetta lander. The box-shaped lander is carried on the side of the orbiter until it arrives at Comet 67P/Churyumov-Gerasimenko. Once the orbiter is aligned correctly, the lander is commanded to self-eject from the main spacecraft and unfolds its three legs, ready for a gentle touchdown at the end of the ballistic descent. On landing, the legs damp out most of the kinetic energy to reduce the chance of bouncing, and they can rotate, lift or tilt to return the lander to an upright position. Immediately after touchdown, a harpoon is fired to anchor the lander to the ground and prevent it escaping from the comet's extremely weak gravity. The minimum mission target is one week, but surface operations may continue for many months.

The Lander science will focus on in- situ study of the composition and structure of the material that constitutes the comet nucleus. Measurement goals include the determination of the elemental, molecular, mineralogical, and isotopic compositions of both the cometary surface and subsurface material. The highest priority will be given to the elemental and molecular determinations, as it is believed that some mineralogical and isotopic measurements can be carried out adequately via the Orbiter science investigations. In addition, properties like near-surface strength, density, texture, porosity, ice phases and thermal properties will be derived. Texture characterization will include microscopic studies of individual grains.

On its way to final destination Rosetta has already flown by 2 asteroids: "2867" Steins and "21" Lutetia. Steins was selected as a scientific target to be observed, and flown by, during the cruise phase of the Rosetta mission. It is a rare, E-type asteroid. Early observations of asteroid Steins were performed using Rosetta's OSIRIS camera in March 2006. Continuous coverage of the asteroid, lasting more than 24 hours was obtained at a phase angle of 41.7 degrees, i.e. larger than the maximum phase angle of Steins (30 degrees) that can be seen from Earth. Several hundred images covering four rotation periods without interruption were acquired. Analysis of the data revealed that the light curve of Steins is double peaked with amplitude of approximately $0.23 \mathrm{mag}$. The rotation period was determined to be $6.052 \pm 0.007$ hours, which confirmed previous measurements obtained from ground.

The OSIRIS camera images show Steins to be an oblate body, resembling a brilliant cut diamond, with dimensions of $6.67 \times 5.81 \times 4.47$ cubic kilometers. Its surface is mostly covered with shallow craters with some of the larger craters being pitted with smaller ones. Analysis of the impact craters reveals a deficit of small craters (those with diameter less than $0.5 \mathrm{~km}$ ) which Keller and his colleagues attribute to surface reshaping as a result of the Yarkovsky-O'Keefe-Radzievskii-Paddack (YORP) effect [59]. The effect would have caused landslides, which filled-in the smaller craters. The YORP effect is a phenomenon that occurs when photons from the Sun are absorbed by a body and reradiated as infrared emission, which carries off momentum as well as heat. The loss of momentum causes a change in the rotation rate of a small body such as an asteroid. The resulting high spin rate of asteroid Steins could have caused material to migrate towards the equator of the asteroid resulting in the distinctive conical shape. This is the first time that the YORP effect has been seen in a main-belt asteroid. Two remarkable features are clearly visible in the images obtained near closest approach: a large, $2.1 \mathrm{~km}$ diameter crater located at the South Pole, and a chain of pits which extend northwards from this crater. Taken together these features suggest that Steins was subject to a big impact which created the large crater and caused fracturing of the asteroid body resulting in it having a rubble pile structure. This type of loosely bound structure is also consistent with the YORP effect hypothesis. Detailed study of the OSIRIS images has also allowed Keller and his colleagues [59] to confirm the nature of Steins as 
an E-type asteroid - the albedo and spectral characteristics are consistent with this classification - and to demonstrate that there is no measurable surface colour variation, which points to a homogeneous composition.

Discovered in Paris by Hermann Goldschmidt in November 1852, asteroid Lutetia has been a cosmic riddle for astronomers. In an attempt to pin down its properties once and for all, ESA's Rosetta spacecraft flew past Lutetia at a distance of $3162 \mathrm{~km}$, at a relative speed of $15 \mathrm{~km} / \mathrm{s}$ on $10 \mathrm{July} 2010$. Situated in the main asteroid belt [60], asteroid Lutetia has been subjected to intense ground-based scrutiny [61] since it was announced as a target for Rosetta in 2004. Initial observations recorded a high albedo, suggesting a high metallic content, and led to the body being classified as an M-type asteroid [62]. The first new photos of the asteroid revealed Lutetia to be a lumpy rock with a potatolike appearance. Its surface is heavily cratered, having suffered many impacts during its 4.5 billion years of existence. As Rosetta drew close, a giant bowl-shaped depression stretching across much of the asteroid rotated into view. The images confirm that Lutetia is an elongated body, with its longest side around $130 \mathrm{~km}[59]$.

Rosetta will be the first spacecraft to orbit a comet's nucleus. It will be the first spacecraft to fly alongside a comet as it heads towards the inner Solar System. Rosetta will be the first spacecraft to examine from close proximity how a frozen comet is transformed by the warmth of the Sun. Shortly after its arrival at Comet 67P/Churyumov-Gerasimenko, the Rosetta orbiter will despatch a robotic lander for the first controlled touchdown on a comet nucleus. The Rosetta lander's instruments will obtain the first images from a comet's surface and make the first in situ analysis to find out what it is made of. On its way to Comet 67P/Churyumov-Gerasimenko, Rosetta has passed through the main asteroid belt, with the option to be the first European close encounter with one or more of these primitive objects. Rosetta will be the first spacecraft ever to fly close to Jupiter's orbit using solar cells as its main power source.

\section{Atmosphere and panspermia}

Although it will be difficult to prove that life has been transported through our Solar System, the chances for the different steps of the process to occur can be estimated. These include (1) the escape process, i.e. the removal to space of biological material that has survived being lifted from the surface to high altitudes; (2) the travel conditions in space, i.e. the survival of the biological material over timescales comparable with the interplanetary passage (3) the entry process, i.e. the non-destructive deposition of the biological material on another planet. Following the identification of meteorites of lunar and Martian origin, we may conclude that the escape of material ranging from small particles up to boulder-size from a planet after the impact of a large asteroid is evidently a feasible process. It is interesting to note that bacterial spores can survive shockwaves produced by a simulated meteorite impacts [63] and huge accelerations [64].

In order to study step (2), the survival of resistant microbial forms in the upper atmosphere and free space, microbial samples have been exposed in situ aboard balloons, rockets and spacecraft such as Gemini, Apollo, Spacelab, the Long-Duration Exposure Facility (LDEF), Foton and Eureca and their responses investigated after recovery [65]. The ESA Microgravity Programme has continued to support such exposure-experiments. A priori, the environment in space seems to be very hostile to life. This is due to the high vacuum, intense radiation of galactic and solar origin, and extreme temperatures. In the endeavour to disentangle the network of potential interactions of the parameters of space, methods have been applied to investigate the impact of each parameter on biological integrity, separately, as well as in combination.

Space vacuum has been considered to be one of the factors that may prevent interplanetary transfer of life because of its extreme dehydrating effect. However, experiments in space have demonstrated that certain micro-organisms survive exposure in space vacuum for extended periods of time, provided they are shielded against the solar UV radiation. Most results are available from spores (a dormant form) of the bacterium Bacillus subtilis. Up to $70 \%$ of the bacterial spores survive short term (e.g. 10 days) exposure to space vacuum. The chances of survival in space are increased if the spores are embedded in chemical protectants such as sugars, or salt crystals, or if they are exposed in thick layers. 
For example, $30 \%$ of subtilis spores survived nearly 6 years in space when embedded in salt crystals and $80 \%$ survived in the presence of glucose.

Solar UV radiation has been found to be the most deleterious factor of space - as tested with dried preparations of viruses, bacterial and fungal spores - with DNA being the critical UV target for lethality. However, about $5 \%$ of a species of the extreme halophile Haloarcula, a salt-loving bacteria, survived a two week space environment during a Foton spaceflight [66].

The radiation field of the Solar System consists of components of galactic and solar origin. It is composed of electrons, alpha-particles and cosmic heavy ions, the latter being the most ionizing and therefore the most deleterious components. The heavy particles of cosmic radiation are conjectured as setting the ultimate limit on the survival of spores in space, because they penetrate even heavy shielding. The maximum time for a spore to escape a hit by a heavy particle has been estimated to be $10^{5}-10^{6}$ years.

During the major part of a hypothetical journey through deep space, microorganisms are confronted with the $4 \mathrm{~K}$ cold of space. Laboratory experiments under simulated interstellar medium conditions point to a remarkably less damaging effect of UV radiation at these low temperatures. Treating B. subtilis spores with three simulated factors simultaneously (UV, vacuum and a temperature of $10 \mathrm{~K}$ ), produces an unexpectedly high survival rate, even at very high UV fluxes. From these data, it has been estimated that, under average conditions in space, spores may survive for hundreds of years [67]. The high survival rate of these spores and the high UV-resistance of micro organisms at the low temperatures of deep space are interesting results. However, travelling from one planet to another, e.g. from Mars to Earth, by chance requires an estimated mean time of several $10^{5}-10^{6}$ years for boulder-sized rocks. Periods of only a few months have been calculated for microscopic particles.

Based on the mean sizes and numbers of meteorites ejected e.g. from Mars and percentages falling on Earth, radiative transfer models for galactic cosmic rays, and laboratory responses of B. subtilis spores and Deinococcus radiodurans cells to accelerated heavy ions, it can be calculated that viable transfer of microbes from Mars to Earth via impact ejecta is possible due to the high number of meteorites and the microorganisms impressive resistance to the dangers of space [68]. Vice-versa, the chances for viable transfers from Earth to Mars are lower.

As for the atmospheric entry, a gneissic impactite inoculated with cryptoendolithic photosynthetic cyanobacteria Chroococcidiopsis sp. was exposed in Earth orbit [69]. After orbiting the Earth for 16 days, the rock entered the Earth's atmosphere and was recovered in Kazakhstan. The heat of entry ablated and heated the rock well below $5 \mathrm{~mm}$, the depth at which light becomes insufficient for photosynthetic organisms (about $5 \mathrm{~mm}$ ), thus killing all of its photosynthetic inhabitants. This experiment shows that atmospheric transit acts as a strong biogeographical dispersal filter to the interplanetary transfer of photosynthesis.

\section{The search for life in the Solar System}

\subsection{Life on Mars and the SNC meteorites}

Mars is a privileged target for the search of extraterrestrial life [70]. The mapping of Mars by Mariner 9, Viking 1 and 2, Mars Global Surveyor and Mars Express, revealed channels and canyons resembling dry river beds. The gamma-ray spectrometer instrument onboard the Mars orbiter Odyssey detected hydrogen, which indicates the presence of water ice in the upper meter of soil in a large region surrounding the planet's South Pole, where ice is expected to be stable [71]. The amount of hydrogen detected indicates 20 to 50 percent ice by mass in the lower layer beneath the top-most surface. The ice-rich layer is about $60 \mathrm{~cm}$ beneath the surface at latitude $60^{\circ} \mathrm{S}$, and approaches $30 \mathrm{~cm}$ of the surface at latitude $75^{\circ} \mathrm{S}$. The presence of water ice in the soil was confirmed by the Phoenix Mars polar lander. The ancient presence of liquid water on the surface of Mars was confirmed by the two American Mars Exploration Rovers, MER, Sprit and Opportunity, and by the presence of sulfates and clays [72]. However, Martian oceans were probably restricted to the very early stages of Martian history. Mars possessed therefore an atmosphere capable of decelerating carbonaceous micrometeorites and chemical evolution may have been possible on the planet. The Viking 1 and 2 lander missions were designed 
to address the question of extant (rather than extinct) life on Mars. Three experiments were selected to detect metabolic activity such as photosynthesis, nutrition and respiration of potential microbial soil communities. Unfortunately, the results were ambiguous since, although "positive" results were obtained, no organic carbon was found in the Martian soil by gas chromatography-mass spectrometry. It was concluded that the most plausible explanation for these results was the presence at the Martian surface of highly reactive oxidants, like hydrogen peroxide, which would have been photochemically produced in the atmosphere. The Viking lander could not sample soils below $6 \mathrm{~cm}$ and therefore the depth of this apparently organic-free and oxidizing layer is unknown. Direct photolytic processes can also be responsible for the degradation of organics at the Martian surface. Although the Viking missions were disappointing for exobiology, in the long run the programme has proved to be extremely beneficial for investigating the possibility of life on Mars.

However, there are Martian rocks on Earth represented by a small group of meteorites, all of igneous (volcanic) origin, called the SNC meteorites (after their type specimens Shergotty, Nakhla and Chassigny) that had comparatively young crystallisation ages, equal to or less than 1.3 billion years. One of these meteorites, designated EETA 79001, was found in Antarctica in 1979. It had gas inclusions trapped within a glassy component. Both compositionally and isotopically, this gas matched the make up of the Martian atmosphere, as measured by the Viking mass spectrometer. The data provide a very strong argument that at least that particular SNC meteorite came for Mars, representing the product of a high energy impact that ejected material into space. There are now 50 SNC meteorites known in total [73]. Two of the SNC meteorites, EETA79001 and ALH84001, supply new and highly interesting information [74]. A subsample of EETA79001, excavated from deep within the meteorite, has been subjected to stepped-combustion. The $\mathrm{CO}_{2}$ release from $200{ }^{\circ} \mathrm{C}$ to $400{ }^{\circ} \mathrm{C}$ suggested the presence of organic molecules. The carbon is enriched in ${ }^{12} \mathrm{C}$, and the carbon isotope difference between the organic matter and the carbonates in Martian meteorites is greater than that observed on Earth. This could be indicative of biosynthesis, although some as yet unknown abiotic processes could perhaps explain this enrichment. David McKay [75] has reported the presence of other features in ALH 84001 that may represent a signature of relic biogenic activity on Mars, but this biological interpretation is now generally rejected $[76,77]$.

Because Mars had a "warm" and wet past climate, it should have sedimentary rocks deposited by running and/or still water on its surface as well as layers of regolith generated by impacts. Such consolidated sedimentary rocks ought therefore to be found amongst the Martian meteorites. However, no such sedimentary material has been found in any SNC meteorite. It is possible that they did survive the effects of the escape acceleration from the Martian surface but did not survive terrestrial atmospheric entry because of decrepitation of the cementing mineral. The 'STONE' experiment, flown by ESA, was designed to study precisely such physical and chemical modifications to sedimentary rocks during atmospheric entry from space. A piece of basalt (representing a standard meteoritic material), a piece of dolomite (sedimentary rock) and an artificial Martian regolith material (80\% crushed basalt and $20 \%$ gypsum) were embedded into the ablative heat shield of Foton 12, which was launched on September 9 and landed on September 24, 1999. Such an experiment had never been performed before and the samples, after their return, were analysed for their chemistry, mineralogy and isotopic compositions by a European consortium. Atmospheric infall modifications are made visible by reference to the untreated samples. The results suggest that some Martian sediment could, in part, survive terrestrial atmospheric entry from space [78].

Even if convincing evidence for ancient life in ALH84001 has not been established, the two SNC meteorites (EETA79001 and ALH84001) do show the presence of organic molecules. This suggests that the ingredients required for the emergence of a primitive life were present on the surface of Mars. Therefore, it is tempting to consider that microorganisms may have developed on Mars and lived at the surface until liquid water disappeared. Since Mars probably had no plate tectonics and since liquid water seems to have disappeared from Mars' surface very early, the Martian subsurface perhaps keeps a frozen record of very early forms of life. NASA has planned a very intensive exploration of Mars and European and Japanese missions are also taking place. Exobiology interests are included, especially in the analysis of samples from sites where the environmental conditions may have been favourable for the preservation of evidence of possible prebiotic or biotic processes. The ESA Manned Spaceflight and Microgravity Directorate convened an Exobiology Science Team to design an integrated suite 
of instruments to search for evidence of life on Mars. Priority was given to the in-situ organic and isotopic analysis of samples obtained by subsurface drilling [79]. The basic recommendations of the Science Team are presently serving as basic guideline for the elaboration of the ESA Exomars mission schedulled for 2016-2018.

\subsection{The EXOMARS program}

Observations from the Planetary Fourier Spectrometer (PFS) [80] on ESA's Mars Express and from very high spectral resolution spectrometers on ground-based telescopes, have detected variable amounts of methane in the atmosphere of Mars [81]. Could this be evidence for life on Mars? International space agencies are planning an ambitious, long-term Mars Robotic Exploration Programme to find a definitive answer to this most enduring question.

Establishing if life ever existed on Mars is one of the outstanding scientific questions of our time. To address this important goal, the European Space Agency (ESA), in cooperation with NASA, has established the ExoMars Programme to investigate the Martian environment and to demonstrate new technologies paving the way for a future Mars sample return mission in the 2020's.

Two missions are foreseen within the ExoMars programme: one consisting of an Orbiter plus an Entry, Descent and Landing Demonstrator Module (EDM) to be launched in 2016 and the other, with a launch date of 2018, consisting of two rovers. Both missions will be carried out in cooperation with NASA.

The ExoMars programme will demonstrate a number of essential flight and in-situ enabling technologies that are necessary for future exploration missions, such as an international Mars Sample Return mission. These include: entry, descent and landing of a payload on the surface of Mars; surface mobility with a Rover; access to the subsurface to acquire samples; and sample acquisition, preparation, distribution and analysis.

The scientific objectives of the joint ESA/NASA ExoMars programme 2016-2018 include: searching for signs of past and present life on Mars, studying the water and geochemical environment as a function of depth in the shallow subsurface, and investigating Martian atmospheric trace gases and their sources.

The Orbiter and EDM will be launched together on an Atlas V 421 and will fly to Mars in a mated configuration. By taking advantage of a direct interplanetary trajectory to Mars the cruise phase can be limited to about 9 months. Upon arrival at Mars the EDM will be ejected on a hyperbolic entry trajectory towards the Red Planet. A few days later the Orbiter will enter orbit followed by a series of manoeuvres including a period of aerobraking operations to finally settle into a circular, 400-km altitude orbit ready to conduct its scientific mission.

The ESA-led Orbiter will perform detailed, remote observations of the Martian atmosphere, searching for evidence of trace gases of possible biological importance, such as methane and its degradation products. Instruments onboard the Orbiter will carry out a variety of studies, including: highly sensitive measurements of trace gases from which the composition of the atmosphere can be determined (this will provide important clues to the possible origin of the gas source, for example, is it of volcanic or biogenic origin?); the measurement and low-resolution imaging of aerosols in the atmosphere, investigation of the temperature variations in the atmosphere, and the mapping of key trace gas species other than methane; high-resolution imaging of gases in order to provide the geological context, if it exists, for sources that have been identified.

Methane $\left(\mathrm{CH}_{4}\right)$ is an organic molecule present in gaseous form in the Earth's atmosphere. Living organisms produce more than $90 \%$ of methane on our home planet. The recent detection of plumes of methane in the northern hemisphere of Mars is of great interest because of its potential biological origin, though other explanations may also be possible. Methane breaks up in the presence of ultraviolet solar radiation. Based on photochemical models and on the current understanding of the composition of the Martian atmosphere, methane has a chemical lifetime of about 300-600 years, which is very short on geological time scales. This implies that the methane that is observed today cannot have been produced 4.5 billion years ago, when the planets formed. So what can explain the presence of this gas on the Red Planet? 
One possibility is a biological origin. The discovery of microbial life 2 to 3 kilometres beneath the surface of the Witwatersrand basin in South Africa led scientists to consider that similar organisms could live, or have lived in the past, below the permafrost layer on Mars. By analogy with Earth, the biological origin of Martian methane could be explained by the existence of micro-organisms, called methanogenes, existing deep under the surface, and producing methane as a result of their metabolism.

If the methane on Mars is biotic, two scenarios could be considered: either long-extinct microbes, which disappeared millions of years ago, have left the methane frozen in the Martian upper subsurface, and this gas is being released into the atmosphere today as temperatures and pressure near the surface change, or some very resistant methane-producing organisms still survive.

An alternative explanation (much more likely) is that the methane is geological in origin. It could be produced, for example, by the oxidation of iron, similar to what occurs in terrestrial hot springs, or in active volcanoes. This gas could have been trapped in solid forms of water, or 'cages', that can preserve methane of ancient origin for a long time. These structures are known as 'clathrate hydrates'.

A geochemical process called serpentinisation could also produce the abiotic methane. Serpentinization is a geological low-temperature metamorphic process involving heat, water, and changes in pressure. It occurs when olivine, a mineral present on Mars, reacts with water, forming another mineral called serpentine, in the presence of carbon dioxide and some catalysts. When certain catalysts are also present, the hydrogen combines with the carbon to form methane. On Mars it is possible to find all these primary elements: olivine, carbon dioxide and some catalysts, but the chemical reaction needs liquid water to occur. This implies that, if the Martian methane comes from serpentinisation, it could be related to subsurface hydrothermal activity.

Concentrations of methane have been observed in 2003 and 2006 in three specific regions of Mars: Terra Sabae, Nili Fossae and Syrtis Major, and data suggest that water once flowed over these areas. Deep liquid water areas below the ice layer would be able to provide a habitat for microorganisms, or a favourable place for the hydro-geochemical production of methane. Further processing in the Martian atmosphere may play an important role that accounts for the observed seasonal variability. Whether geochemical or biochemical in origin, the variation in concentrations of methane that has been measured indicates that Mars could still be active today.

The 2018 mission is a NASA-led mission and includes two rovers, one European and the other American. Both rovers will be transported in the same aeroshell and will be delivered to the same site on Mars. The ESA ExoMars Rover will carry a drill and a suite of instruments dedicated to exobiology and geochemistry research.

In attempting to define an effective strategy to search for carbon-based life on Mars, a useful approach is to initially ponder separately the issues of past and present life detection; and subsequently, to look for a common thread that may suggest a way to address both cases.

If life ever arose on the Red Planet, it probably did when Mars was warmer and wetter, during its initial half to one billion years. Conditions then were similar to those on the young Earth. Therefore, one may reasonably expect that, likewise, life emerged on early Mars, and that microbes quickly became a global phenomenon. Nevertheless, there is inevitably a large measure of chance involved in finding convincing evidence of ancient life forms. On our planet's surface, the permanent presence of running water, atmospheric oxygen, and life itself quickly erases all traces of any exposed, dead organisms. The only opportunity to detect them is to find their biosignatures encased in a protective environment, i.e. in suitable rocks. However, since high-temperature metamorphic processes and plate tectonics have resulted in the reforming of most ancient terrains, it is very difficult to find rocks on Earth older than 3 Gyr in good condition. The ensuing chemical and isotopic degradation of many putative bacterial fossils makes their reliable identification far from trivial.

A further complication is that a range of inorganic processes is known to result in mineral structures closely resembling simple biological shapes. This issue lies at the heart of a heated debate among palaeobiologists. Two recent examples that have attracted much attention are the early Archean rock specimens, obtained from the Pilbara Region in Western Australia, claimed to contain Earth's oldest cyanobacteria-like fossils to date, and the Martian meteorite ALH84001, whose alleged fossil microorganisms were seen worldwide in 1996. The difficulty is that, in essence, we are looking for the exiguous remnants of minuscule, unshelled, uncompartmentalised beings whose fossilized forms can be confused with tiny mineral precipitates. It is therefore doubtful that the living origin of ancient 
candidate microfossils may be accurately established on the basis of their morphology alone. Although important, comparative anatomy by itself cannot be relied upon to provide sufficient proof.

Another useful clue may lie in the isotopic signature of carbon. Many life processes favor the assimilation of the light isotope, ${ }^{12} \mathrm{C}$, over that having an extra neutron, ${ }^{13} \mathrm{C}$. This gives rise to a higher concentration of ${ }^{12} \mathrm{C}$ in living cells relative to the one found in the surrounding dead environment. For instance, the enzymatic uptake of carbon during photosynthesis can result in a ${ }^{12} \mathrm{C} /{ }^{13} \mathrm{C}$ ratio significantly higher than the one used as standard for terrestrial abiotic material. Consequently, provided they can be isolated, carbon residues stemming from previously living matter may be recognised by their ${ }^{12} \mathrm{C}$ enrichment. However, the heating of rocks to high temperatures quickly converts any original cell material to graphite, degrading this signal and making it hard to interpret [82]. For a useful interpretation of isotope biosignatures, a detailed understanding of the sources and sinks, as well as their temporal evolution, is crucial.

Some compounds synthesised by living organisms are so stable that they can last for billions of years after the parent cells have died and decomposed. It is not the whole molecule that survives, but rather the backbone of carbon atoms with its distinctive geometry. Typical examples are amino acids, the lipids that comprise cell walls, and some important pigments, such as bacteriochlorophyll and chlorophyll that absorb light to power photosynthesis in bacteria and plants. These telltale molecules are very common on our planet and can constitute very reliable biomarkers. Identifying one of them could prove as informative as finding a dinosaur bone.

Regrettably, a major problem with the study of biomarkers is that many decompose when exposed to temperatures greater than $200{ }^{\circ} \mathrm{C}$. As already discussed, most Archean rocks on Earth have been heated beyond this value. Mars, on the other hand, has not suffered such widespread tectonic activity. Long-lasting volcanism seems to have concentrated mainly on large provinces, such as the Tharsis and Elysium regions. This would imply that rock formations from the earliest period of Martian history, which have not been exposed to high-temperature recycling, may exist. Consequently, wellpreserved ancient biomarkers may still be accessible for analysis. As already mentioned in Chapter 4.2.1, most important molecular building blocks of life can exist in left- and right-handed configurations called enantiomers which, like a pair of gloves, are mirror images of one another. On Earth all living organisms use one enantiomer only: left-handed in the case of amino acids and right-handed for sugars. This property of homochirality is essential for an efficient metabolism. Key life processes, such as protein synthesis and gene transcription, rely on amino acids and sugars having the correct spatial conformation to "shake hands" at molecular level with their counterparts. Conversely, synthetic chemicals prepared in the laboratory exhibit equal abundances of both right- and left-handed enantiomers, such a mixture is said to be racemic. Homochirality probably constitutes the most reliable indicator of the biological vs. abiotic origin of organic molecules. Surely, testing for homochirality becomes crucial when searching for life. However, as in the previous methods outlined, unfortunately also this one suffers when the sample is exposed to high temperatures or wet conditions for extended periods.

Summarizing, the best chance to find signatures of ancient life on Mars is in the form of chemical biomarkers and fossil communities, either preserved underground or encased within surface rocks. A few life-detection methods, by no means exhaustive, were presented to illustrate how important it is to use complementary techniques that, combined, give more credence to the proposition of a sample's biological potential. Several independent lines of evidence are required to construct a compelling case. ExoMars must therefore pursue a holistic search strategy, attacking the problem from multiple angles, including geological and environmental investigations (to characterize potential habitats), visual examination of samples (morphology), and spectrochemical composition analyses.

The science objectives of the ExoMars Rover, in order of priority, are: to search for signs of past and present life on Mars; to characterise the water/geochemical environment as a function of depth in the shallow subsurface.

The ExoMars Rover mission will pursue one of the outstanding questions of our time by attempting to establish whether life ever existed, or is still active on Mars today. The Rover will carry a comprehensive suite of analytical instruments dedicated to exobiology and geochemistry research: the Pasteur payload. The Rover will travel several kilometres searching for signs of past and present life, 
collecting and analysing samples from within rocky outcrops and from the subsurface, down to a depth of $2 \mathrm{~m}$.

The ExoMars rover will search for two types of life-related signatures: morphological and chemical. This will be complemented by an accurate determination of the geological context. Morphological information related to biological processes [83] may be preserved on the surface of rocks. Possible examples include the bio-mediated deposition of sediments, fossilised bacterial mats, stromatolithic mounds, etc. Such studies require mobility and an imaging system capable of covering a range of distances from the metre scale down to sub-millimetre resolution (to discern micro-textural information in rocks).

An effective chemical identification of biomarkers requires access to well-preserved organic molecules. Because the Martian atmosphere is more tenuous than Earth's, three important physical agents reach the surface of Mars with adverse effects for the long-term preservation of biomarkers: the ultraviolet (UV) radiation dose is higher than on our planet and will quickly damage potential exposed organisms or bio-molecules; UV-induced photochemistry is responsible for the production of reactive oxidant species that, when activated, can also destroy biomarkers; the diffusion of oxidants into the subsurface is not well characterised and constitutes an important measurement that the mission must perform [84].

The ionising radiation penetrates into the uppermost metres of the planet's subsurface. This causes a slow degradation process that, over many millions of years, can alter organic molecules beyond the detection sensitivity of analytical instruments. The ionising radiation effects are depth dependent: the material closer to the surface is exposed to a higher dose than that buried deeper.

A major goal of ExoMars is to study ancient (older than 3 billion years) sedimentary rock formations and evaporitic deposits. However, it is only trapped in the subsurface for long periods that the record of early Martian life, if it ever existed, is likely to escape radiation and chemical damage. Studies show that a sub-surface penetration in the range of $2 \mathrm{~m}$ is necessary to recover well-preserved organics from the very early history of Mars. Additionally, it is essential to avoid loose dust deposits distributed by aeolian transport. While driven by the wind, this material has been processed by UV radiation, ionising radiation, and potential oxidants in the atmosphere and on the surface of Mars [85]. Any organic biomarkers would be highly degraded in these samples.

For all the above reasons, the ExoMars drill will be able to penetrate and obtain samples from well-consolidated (hard) formations, at various depths, down to $2 \mathrm{~m}$ [86].

Being liquid water a prerequisite for active life, good candidate locations to look for microfossils are terrains occupied by long lasting bodies of water during Mars's early history. For example, within ancient lacustrine or marine sedimentary rocks that accumulated rapidly, where subsequent diagenesis did not obliterate the original texture and compositional evidence [87], isotopic, organic, and mineralogical, of the deposition environment. Some promising sites have been identified through the analysis of Mars Global Surveyor, Odyssey images, and Mars Express images.

For organisms to have emerged and evolved, liquid water must have been present on Mars. Without it, most cellular metabolic processes would not be possible. In the absence of water, life either ceases or slips into a quiescent mode. Hence, the search for extinct or extant life automatically translates into a search for liquid water-rich environment, past or present [88].

The strategy to find traces of past biological activity rests on the assumption that any surviving signatures of interest will be preserved in the geological record, in the form of buried/encased remains, organic materials, and fossil communities.

Similarly, because current Martian surface conditions are hostile to most known organisms, also when looking for signs of extant life, the search methodology should focus on investigations in protected niches: underground, in permafrost, or within surface rocks. Therefore, the same sampling device and instrumentation can adequately serve both types of studies. The biggest difference is due to location requirements. In one case, the interest lies in areas occupied by ancient bodies of water over many thousands of years. In the other, the emphasis is on water-rich environments close to the surface and accessible to our sensors today. For the latter, the presence of permafrost alone may not be enough. Permafrost in combination with a sustained heat source, probably of volcanic or hydrothermal origin, may be necessary. Such warm oases can only be identified by an orbital survey of the planet. In the next few years, a number of remote sensing satellites, like ESA's Mars Express and NASA's Mars 
Reconnaissance Orbiter (MRO), will determine the water/ice boundary across Mars and may help to discover any such warm spots. If they do exist, they would be prime targets for missions like ExoMars.

On Earth, microbial life quickly became a global phenomenon [89]. If the same explosive process occurred on the young Mars, the chances of finding evidence of past life may be good. Even more interesting would be the discovery and study of life forms that have successfully adapted to modern Mars, such as the cryptoendolithic communities discussed above. However, this presupposes the prior identification of geologically suitable, life-friendly locations where it can be demonstrated that liquid water still exists, at least episodically throughout the year. For these reasons, the first exobiology science team advised ESA to focus mainly on the detection of extinct life, but also to build enough flexibility into the mission design to be able to target sites with potential for present life.

If any organic compounds are detected on Mars, it will be important to show that they were not brought from Earth. The only way to reliably demonstrate that the Rover is free from contaminants is to perform an initial measurement run using a blank sample. For this reason, the ExoMars rover will carry a number of blank calibration samples. Upon landing, one of the first science actions will be for the drill to pass a blank sample to the analytical laboratory. After performing a full investigation, the results should indicate 'no life' and 'no organics'. Failure to obtain this first negative reading could invalidate any search-for-life findings.

Throughout its nominal 180-sol mission, the ExoMars Rover will be able to perform 6 Experiment Cycles (each involving the acquisition and analysis of a surface and a subsurface sample at an individual site), and 2 Vertical Surveys (each vertical survey consists of obtaining and analysing five subsurface samples at $50 \mathrm{~cm}$ depth increments, from 0 to $200 \mathrm{~cm}$, to study variations with depth at one location).

\subsection{Europa}

Europa appears as one of the most enigmatic of the Galilean satellites. With a mean density of about $3.0 \mathrm{~g} \mathrm{~cm}^{-3}$, the Jovian satellite should be dominated by rocks. Ground-based spectroscopy, combined with gravity data, suggests that the satellite has a kilometers thick icy crust and a rocky interior. The Voyager images showed very few impact craters on Europa's surface, indicating recent, and probably continuing, resurfacing by cryovolcanic and tectonic processes. Images of Europa's surface taken by the Galileo spacecraft show surface features, iceberg-like rafted blocks, cracks, ridges and dark bands, which are consistent with the presence of liquid water beneath the icy crust. Data from Galileo's Near-Infrared Mapping Spectrometer show hydrated salts which could be evaporites. The most convincing argument for the presence of an ocean of liquid water comes from Galileo's magnetometer. The instrument detected an induced magnetic field within Jupiter's strong magnetic field. The strength and response of the induced field require a near-surface, global conducting layer, most likely a layer of salty water $[90,91]$.

If liquid water is present within Europa, it is quite possible that it includes organic matter derived from thermal vents. Terrestrial-like prebiotic organic chemistry and primitive life may therefore have developed in Europa's ocean. If Europa maintained tidal and/or hydrothermal activity in its subsurface until now, it is possible that microbial activity is still present. Thus, the possibility of an extraterrestrial life present in a subsurface ocean of Europa must be seriously considered. The most likely sites for extant life would be at hydrothermal vents below the most recently resurfaced area. To study this directly would require making a borehole through the ice in order to deploy a robotic submersible. On the other hand, biological processes in and around hydrothermal vents could produce biomarkers that would be erupted as traces in cryovolcanic eruptions and thereby be available at the surface for in situ analysis or sample return. Mineral nutrients delivered through cryovolcanic eruption would make the same locations the best candidates for autotrophic life.

\subsection{Titan}

Titan is the only object in the solar system to bear a resemblance to our own planet in terms of atmospheric pressure ( 1.5 bar) and carbon/nitrogen chemistry. Titan's atmosphere was revealed mainly 
by the Voyager 1 mission in 1980, which yielded the bulk composition (90\% molecular nitrogen and about 1-8\% methane). A great number of trace constituents were also observed in the atmosphere in the form of hydrocarbons (acetylene, ethane, ethylene, etc.), nitriles $\left(\mathrm{HCN}, \mathrm{C}_{2} \mathrm{~N}_{2}, \mathrm{HC}_{3} \mathrm{~N}\right)$ and oxygen compounds $\left(\mathrm{CO}\right.$ and $\left.\mathrm{CO}_{2}\right)$, attesting to a very active organic chemistry running in the atmosphere. The ISO satellite has detected tiny amounts of water vapour in the higher atmosphere, but Titan's surface temperature $(94 \mathrm{~K})$ is much too low to allow the presence of liquid water. However, these molecules might initiate an exotic carbon-based life in the hydrocarbon oceans present on Titan's surface.

\subsubsection{The Cassini-Huygens mission}

Cassini-Huygens is a joint NASA/ESA/ASI mission to explore Saturn, Titan and the other moons of the Saturnian system. The mission has two distinct elements: the Cassini orbiter and the Huygens probe. NASA's Jet Propulsion Laboratory, with the Italian Space Agency (ASI) contributing its highgain antenna and other radio subsystem equipment under a bilateral NASA/ASI agreement, built the Cassini orbiter. Huygens, and the associated communications equipment on the orbiter, was built by ESA.

The Cassini-Huygens spacecraft was launched at 08:43 UT on 15 October 1997 by a Titan IVBCentaur rocket from Cape Canaveral Air Force Station in Florida, USA. The 5.6-ton spacecraft was too heavy to be injected into a direct trajectory to Saturn, so the interplanetary voyage of about 6.7 years includes gravity-assist maneuvers at Venus, Earth and Jupiter. On 1 July 2004, the CassiniHuygens spacecraft entered the Saturnian system marking the start of its 4-year nominal mission. On 25 December 2004, towards the end of the third orbit around Saturn, the Cassini orbiter jettisoned the Huygens probe for its 20-day journey to Titan. On 14 January 2005, the Huygens probe successfully entered Titan's upper atmosphere and descended under parachute to the surface. The descent phase lasted around 2 hours 27 minutes with a further 1 hour 10 minutes on the surface. Throughout the mission data was collected from all instruments providing a detailed picture of Titan's atmosphere and surface. During each Titan flyby, the Orbiter has performed a set of in orbit and remote sensing observations of the surface, the atmosphere and the plasma environment. The detailed atmosphere descent data set acquired by the Probe and the global coverage that provided by the Orbiter observations during targeted flybys have provided a unique wealth of new scientific information.

NASA's Cassini successfully completed its first close flyby of a moon of Saturn on Friday, June 11, 2004, when the spacecraft captured images of the moon Phoebe. Detailed images revealed a heavily cratered surface that has astronomers debating the satellite's origins. The most detailed image released so far shows a tiny world riddled with ancient pockmarks, but with great variations of surface brightness. Phoebe in general is very dark, but close inspection revealed areas so bright that they were washed out in the picture. The sharp clear images showed many geological features. Researchers believe closer inspection of the pictures of Phoebe could reveal it to be indeed a Kuiper Belt object [92].

The Cassini orbiter has continued its exploration of the Saturn's system over the course of 75 orbits around Saturn lasting four years. It has made repeated close flybys of Titan, both to gather data about the moon and for gravity-assist orbit changes that have enabled it to make a tour of Saturn's satellites, explore the planet's magnetosphere and obtain views of Saturn's higher latitudes.

The overall scientific objectives of the mission at Titan are to: determine abundance of atmospheric constituents (including noble gases); establish isotope ratios for abundant elements; constrain scenarios of formation and evolution of Titan and its atmosphere; observe vertical and horizontal distributions of trace gases; search for more complex organic molecules; investigate energy sources for atmospheric chemistry; model the photo-chemistry of the stratosphere; study formation and composition of aerosols; measure winds and global temperatures; investigate cloud physics, general circulation and seasonal effects in Titan's atmosphere; search for lightning discharges; determine the physical state, topography and the composition of the surface; infer the internal structure of the satellite; investigate the upper atmosphere, its ionization, and its role as a source of neutral and ionized material for the magnetosphere of Saturn.

The $14^{\text {th }}$ of January 2005 represents a milestone in space exploration. At around 11:30 UTC the Huygens probe successfully landed on the surface of Titan, making it the first probe to land on an 
object in the outer Solar System. The Huygens mission officially started at around 09:06 UTC when the Huygens probe reached the predetermined interface altitude of $1270 \mathrm{~km}$ above the surface of Titan. Prior to this, two crucial events took place that marked a transition from the cruise phase to operations phase: 04:44 UTC Mission Timer Unit (MTU) activated the Huygens probe; 07:02 UTC Cassini turned to point the HGA at the landing site in order to communicate with the probe. Communications did last for a maximum of $4 \mathrm{~h} 36$ minutes: after that, Huygens has passed over the horizon relative to Cassini and contact was lost. Alongside the spacecraft instrument readings the descent of Huygens was tracked by radio telescopes around the world, which monitored the carrier signal sent by a transmitter activated when the first parachute deployed. The confirmation of the detection of this signal by the Green Bank 110m dish at around 10:30 UTC was the first indication that the mission had gone to plan.

The probe descended through haze until an altitude of about $30 \mathrm{~km}$ above the surface. At an altitude of $700 \mathrm{~m}$ above the surface the descent lamp was activated. The purpose of this lamp was to not to illuminate the landing site, the light levels on the surface of Titan are roughly 1000 times less than sunlight and 1000 times stronger than a full moon, but to provide a monochromatic light source and enable scientists to accurately determine the reflectivity of the surface.

The surface phase of the mission lasted $1 \mathrm{~h} 10$ minutes, considerably longer than had been anticipated. There were three main reasons for this: there was no failure on the batteries so all five could be used to power the probe; the landing on the surface was soft, meaning no damage was done to the probe.

The Huygens probe landed near a bright region now called Adiri. Images sent back to Earth showed light hills cut by dark riverbeds that empty into a dark plain. Before the Huygens probe arrived, scientists believed that this dark plain could be a lake or at least a muddy material. But Huygens actually landed in this dark plain, revealing a surface of gravel and small boulders made of water ice. Scientists believe it only rains occasionally on Titan, but that the methane rains are extremely fierce when they come, carving channels in the surface similar to those observed in arid regions on Earth.

Only a small number of impact craters have been discovered. This suggests that, like Earth, Titan's surface is constantly being resurfaced by erosion, caused by both flowing liquid and wind. Cryovolcanism may be another resurfacing mechanism, with the lava consisting of a fluid mixture of water and possibly ammonia, believed to be expelled from volcanoes and hot springs. Some surface features, such as lobe-shaped flows, appear to be volcanic in origin, giving further support to the cryovolcanism theory. In addition, volcanism is now believed to be a significant source of methane in Titan's atmosphere, since there are no oceans of hydrocarbons as had been hypothesized previously [93].

The main messages were: i) nitrogen and methane are the main constituents of the atmosphere; ii) the isotopic ratio ${ }^{12} \mathrm{C} /{ }^{13} \mathrm{C}$ suggests a permanent supply of methane in the atmosphere; iii) the surface is "wetted" by liquid methane and rich in organics (cyanogen, ethane); iv) the presence of ${ }^{40} \mathrm{Ar}$ suggests the existence of internal geological activity [94].

Dunes cover large areas of the surface. The dunes may be made of hydrocarbon particulate material, or possibly solid accumulations of hydrocarbons. Whatever their nature, the dunes contain less water ice than other parts of Titan's surface, and might consist of haze particles produced in the atmosphere rather than being composed of the equivalent of sand produced by erosion [95].

The existence of oceans or lakes of liquid methane on Saturn's moon Titan was predicted more than 20 years ago. Radar, imaging and spectral data from Titan flybys have provided convincing evidence for large bodies of liquid near Titan's north and south poles [96,97]. With Titan's colder temperatures and hydrocarbon-rich atmosphere, these lakes and seas contain a combination of liquid methane and ethane (both hydrocarbons), not water. Ongoing monitoring of the lakes will tell us more about Titan's methane cycle and methane table, and if these are subject to seasonal change [98]. Radar mapping and gravity data suggest that Titan has an interior ocean of liquid water and ammonia, perhaps $100 \mathrm{~km}$ (60 miles) below the surface [99].

Cassini-Huygens arrived at Saturn during the planet's northern winter and southern summer (roughly the equivalent of mid-January on Earth). During Cassini's four-year nominal mission, as Saturn has moved towards its vernal equinox (which it reached in August 2009), changes in Titan's cloud distribution have been observed that may be due to the advancing seasons. In the early part of the Cassini mission, large convective cloud systems were observed at the south (summer) pole, but 
these have become less common, while long streaks of clouds have been seen progressively further north. Titan's detached haze layer may also be subject to seasonal changes that push its altitude higher.

The Cassini-Huygens mission, using wavelengths ranging from ultraviolet to radio, continues to reveal more of Titan and answer long-held questions regarding Titan's interior, surface, atmosphere, and the complex interaction with Saturn's magnetosphere. While many pieces of the puzzle are yet to be found, with each Titan flyby comes a new data set that furthers our understanding of this fascinating world.

Enceladus was known to be geologically active since July 2005, when Cassini instruments discovered the presence of geysers at the south pole of this small icy moon. It had long been suspected that Enceladus might be active. The Voyager 2 encounter with the satellite in 1982 established that its geometric albedo is startlingly high, at about 1.0, a number that is consistent with fresh snow or ice. Recent results from the Hubble Space Telescope yield a value of 1.4 for the geometric albedo. Voyager 2 imaged sizable regions of crater-free areas thought to have been resurfaced within the last $1 \mathrm{~Gy}$, but other parts of the satellite were older $(\sim 3.9 \mathrm{~Gy})$ and heavily cratered. Moreover, all regions of the satellite, whether young or old, exhibited uniformly high albedos, implying that the entire satellite is coated with a ubiquitous fresh material. Dynamic models have proposed that Enceladus is the source of the E-ring, which is most dense at the satellite's orbit and extends out to the distance of Titan.

Cassini has executed several close passes of Enceladus. The first flyby, which occurred on February 17, 2005 with a minimum approach distance of 1175 kilometers, focused on the equatorial region of the sub-Saturn/trailing hemispheres. The Imaging Science Subsystem (ISS) revealed a world scarred by extensive tectonic activity with both extensional and compressional features. Magnetometer data showed a draping of Saturn's magnetic field lines around the moon, which suggested the presence of an atmosphere. However, a stellar occultation observed by the Ultraviolet Imaging Spectrometer (UVIS) showed no sign of an atmosphere. Infrared spectra from the Visual Infrared Mapping Spectrometer (VIMS) detected no surface components other than water ice. During the second flyby, on March 9, 2005, Cassini came within 500 kilometers of Enceladus and observations concentrated on the equatorial region of the anti-Saturn/trailing hemisphere. This encounter revealed the diverse regions on Enceladus in striking detail: large complex networks of ridges and troughs coexisted with ancient cratered plains. The magnetometer measured a signature in addition to the one seen in February, one that possibly indicated an induced or intrinsic magnetic field. Based on these results which could imply a liquid sub-surface region, the Cassini Project moved the closest approach distance of the July 14 encounter from 1000 kilometers down to 175 kilometers.

The July 2005 observations of the South Polar Region by the remote sensing instruments, a stellar occultation experiment and the close flyby distance over the South Pole allowed in-situ measurements by particles and fields instruments. The observations provided multi-instrument evidence of geologic activity on Enceladus.

The fourth close Enceladus flyby, on March 12, 2008, focused on plume measurements by fieldsand-particles instruments. By flying deeper into the plume than on previous flybys, the Ion and Neutral Mass Spectrometer (INMS) was able to obtain more sensitive measurements of the composition of the plume. In addition, the Composite Infrared Mapping Spectrometer (CIRS) mapped the temperatures at the south pole while Enceladus was in eclipse and found that the highest temperatures are found along the tiger stripes, where most of the jets are also seen by ISS.

The fifth close Enceladus flyby, on Aug. 11, 2008, provided the highest-resolution visible images of the south polar tiger stripes of the mission $(\sim 7 \mathrm{~m} /$ pix $)$. The images suggest differences between currently active local regions and places that may have been formerly active. Interestingly, the flyby was designed for (and thus the spacecraft was oriented to optimize) remote sensing measurements; the INMS measured a very strong signal from the plume species [100].

The major question that has emerged is why geologic activity exists at all on this small world. Other active satellites - Io $(\mathrm{R}=1810 \mathrm{~km})$ and Triton $(\mathrm{R}=1350 \mathrm{~km})$ - are far larger. Even with a bulk density of $1.6 \mathrm{gm} / \mathrm{cm} 2$, heat produced in Enceladus' core from radioactive decay would have long since peaked and dissipated. One mechanism that works marginally is tidal heating from eccentricities excited by the orbital resonance between Enceladus and Dione. Even if a sufficient heat source can be found, the question still remains: why is the activity concentrated at the South Pole? These Cassini 
results have strong implications for models of planetary interiors and the role of tidal heating and dissipation [101, 102].

Cassini completed its initial four-year mission to explore the Saturn System in June 2008. Now, the healthy spacecraft is working overtime on the Cassini Equinox Mission, seeking answers to new questions raised in Cassini's first years at Saturn.

The mission's extension, through September 2010, is named for the Saturnian equinox, which occurs in August 2009 when the sun will shine directly on the equator and then begin to illuminate the northern hemisphere and the rings' northern face. Cassini will observe seasonal changes brought by the changing sun angle on Saturn, the rings and moons, which were illuminated from the south during the mission's first four years. Many repeat visits are planned for Cassini at the moons Titan and Enceladus, both important targets of the Equinox Mission.

Cassini's continued journey carries it throughout the huge sphere of magnetic activity that surrounds Saturn. A major discovery has been that water ice jets from Enceladus play a major role in Saturn's magnetosphere. Water from the jets loads up the magnetosphere, influencing radio and auroral activity, and even causing changes in the rotation of the magnetic field itself. The Equinox mission is studying these phenomena in unexplored areas of the magnetosphere and probing the links to Enceladus in addition to connections with other moons.

\section{The search for life beyond the Solar System}

\subsection{The search for rocky Earth-like exoplanets}

Apart from abundant hydrogen and helium, about 125 interstellar and circumstellar gaseous molecules have currently been identified in the interstellar medium. It is commonly agreed that the catalog of interstellar molecules represents only a fraction of the total spectrum of molecules present in space, the spectral detection being biased by the fact that only those molecules possessing a strong electric dipole can be observed. Among these molecules, 110 contain carbon. Therefore, the interstellar medium tells us that organic chemistry is universal. What about liquid water? New planets have been discovered beyond the Solar System [103,104]. On October 6, 1995, the discovery of an extrasolar planet was announced by Michel Mayor and Didier Queloz. The planet orbits an eight billion year old star called 51 Pegasus, forty-two light years away within the Milky Way. The suspected planet takes just four days to orbit 51 Pegasus. It has a surface temperature around $1,000{ }^{\circ} \mathrm{C}$ and a mass about 0.5 the mass of Jupiter. As of September 2010, 490 exoplanets have been observed [105]. Astronomers using ESO's HARPS telescope have discovered a planetary system containing at least five planets, orbiting the Sunlike star HD 10180. Two other planets may be present, one of which would have the lowest mass ever found. This would make the system similar to our Solar System in terms of the number of planets.

\subsubsection{The COROT mission}

The European space telescope COROT is the first mission capable of detecting rocky planets, several times larger than Earth, around nearby stars. It was launched on 27 December 2006. COROT uses its telescope to monitor closely the changes in a star's brightness that comes from a planet crossing in front of it. While it is looking at a star, this spacecraft is also able to detect 'starquakes', acoustical waves generated deep inside a star that send ripples across a star's surface, altering its brightness. The exact nature of the ripples allows astronomers to calculate the star's precise mass, age and chemical composition. This technique is known as asteroseismology and ESA's Solar and Heliospheric Observatory (SOHO) has been taking similar observations of the Sun for years. The COROT data is therefore essential to compare the Sun with other stars.

The payload of the COROT satellite consists of a telescope, two cameras - one for each of the two mission objectives (exoplanet search and asteroseismology), and the on-board computer processors. The telescope is made out of two parabolic mirrors, having a 1.1-metre focal length. The field of view is a square of $2.8 \times 2.8^{\circ}$, half for the seismology mission, the other half for the exoplanet mission. A 
prism, used by the telescope for certain aspects of the mission, will separate colours, enabling scientists to study stellar activity during a planetary transit.

The mission has detected several small planets including COROT-7b, rocky and having 1.7 times the size of the Earth, but too hot to host life (about $1000^{\circ} \mathrm{C}$ ), and Corot-9b with a temperature possibly ranging between $-23{ }^{\circ} \mathrm{C}$ and $157^{\circ} \mathrm{C}$.

More than 400 exoplanets have been discovered so far and 70 of them have been found by the 'transit' method. A transit is a kind of eclipse and occurs when a celestial body passes in front of its host star and blocks some but not all of the star's light. This temporarily dims the apparent brightness of the star and enables the planet's mass, diameter, density and temperature to be deduced. The time between similar transits gives the orbital period of the planet.

Corot-9b is the first transiting planet to have both a longer period and a near-circular orbit. Its orbit is slightly elliptical but at closest approach to its parent star it reaches a distance of 54 million kilometres. Although that is only about the distance of Mercury in our Solar System, it is by far the largest orbit of any transiting planet found so far. Corot-9b has a radius around 1.05 times that of Jupiter but only $84 \%$ of the mass. This leads to a density of $0.90 \mathrm{~g} / \mathrm{cc}$, or $68 \%$ that of Jupiter.

\subsubsection{Detecting extrasolar life}

Extrasolar life, i.e. life on a planet of a stellar system other than our own, will not be accessible to space missions in the forseable future. The formidable challenge of detecting distant life must therefore be tackled by astronomers and radioastronomers. The simultaneous detection of water, carbon dioxide and ozone (an easily detectable telltale signature of oxygen) in the atmosphere would constitute the most convincing biomarker but not an absolute proof. Other anomalies in the atmospheres of rocky Earth-like exoplanets, such as the presence of methane, could indicate the presence of an extrasolar life. European astrophysicists are proposing the construction of a flotilla of four free-flying spacecrafts, each containing a 3 metre diameter infrared telescope to search for signs of life on terrestrial-like planets. The mission, called Darwin [106] is presently under study at ESA. Finally, the detection of an unambiguous electromagnetic signal via the SETI program would obviously be an exciting event [107].

\section{Conclusion}

Terrestrial atmosphere and the correlated hydrosphere, play a crucial role in modern biology and the love story probably started with the origin of life. It is therefore legitimate to search for liquid water when searching for extraterrestrial life. Even if somewhat egocentric, and therefore restrictive, searching for liquid water is the easiest way to recognise any extraterrestrial life. Concerning the possible existence of another water-based life, the early histories of Mars and Earth clearly show some similarities. The existence of large valley networks and different channels strongly suggests that liquid water was once stable on the surface of Mars. If organic molecules were brought to Mars by cometary grains or meteorite impacts, then an aqueous organic chemistry might have developed on Mars until liquid water disappeared about 3.5 billion years ago. The search for organic molecules and fossilised primitive life below the Martian surface is probably the most fascinating prospect for Mars exploration. Europa's ice shell might be separated from the silicate interior by a liquid water layer. If liquid water is present within Europa, it is quite possible that it includes organic matter derived from thermal vents. Terrestrial-like prebiotic organic chemistry and primitive life may therefore have developed in Europa's ocean. Other "biogenic" liquid must not be excluded. For instance, Saturn moon Titan hosts an ocean of methane and ethane and perhaps an exotic form of life which might be very difficult to identify. Searching for a second genesis of a water-based life on another celestial body will be one of the most exciting challenges for the coming decades. 


\section{References}

1. D. L. Pinti, in Lectures in Astrobiology, pp. 83-112 (M. Gargaud, B. Barbier, H. Martin, J. Reisse, editors, Springer-Verlag, Heidelberg, 2005)

2. J.F. Kasting, L.L. Brown, in The molecular origins of life: assembling pieces of the puzzle, pp. 35-56 (A. Brack, editor, Cambridge University Press, Cambridge, 1998)

3. D. Catling, J. F. Kasting, in Planets and Life, pp. 91-116 (W. T. Sullivan III, J. A. Baross, editors, Cambridge University Press, 2007)

4. J.C.G. Walker, Origins Life Evol. Biosphere 16, 117 (1985)

5. R.M. Canup, E. Asphaug, Nature 412, 708 (2001)

6. J.C.G. Walker, Origins Life Evol. Biosphere 16, 117 (1985)

7. F. Robert, Science 293, 1056 (2001)

8. M. Maurette, Micrometeorites and the mysteries of our origins (Springer Berlin /Heidelberg, 2006)

9. J.D. Bernal, Proc. Roy. Soc. London 357A, 537 (1949)

10. A. Brack, Chem. Biodivers. 4, 665 (2007)

11. Y. Ogata, E.-I. Imai, H. Honda, H. K. Hatori, K. Matsuno, Origins Life Evol. Biosphere 30, 527 (2000)

12. W. Bains, Astrobiology 4, 137 (2004)

13. J.A. Baross, S.E. Hoffman, Orig. Life Evol. Biosphere 15, 327 (1985)

14. N.G. Holm, E.M. Andersson, in The molecular origins of life: assembling pieces of the puzzle, pp. 86-99 (A. Brack, editor, Cambridge University Press, Cambridge, 1998)

15. N.G. Holm, E.M. Andersson, Astrobiology 5, 444 (2005)

16. N.G. Holm, J.-L. Charlou, Earth Planet. Sci. Lett. 19, 1 (2001)

17. C. Ponnamperuma, A. Shimoyama, E. Friebele, Origins Life 12, 9 (1982)

18. A. Brack, in Handbook of Clay Science, pp. 385-398 (F. Bergaya, B.K.G. Theng, G. Lagaly, editors, Elsevier Science, 2006)

19. A. Negron-Mendoza, S. Ramos-Bernal, F.G. Mosqueira, in Astrobiology: emergence, search and detection of life, pp. 214-233 (V.A. Basiuk editor, American Scientific Publishers, Stevenson Ranch, California, USA, 2010)

20. G. Wächtershäuser, Proc. Natl. Acad. Sci. USA 91, 4283 (1994)

21. G. Wächtershäuser, in The molecular origins of life: assembling pieces of the puzzle, pp. 206-218 (A. Brack, editor, Cambridge University Press, Cambridge 1998)

22. G. Wächtershäuser, Chem. Biodiv. 4, 584 (2007)

23. W. Heinen, A.M. Lauwers, Origins Life Evol. Biosphere 26, 131 (1996)

24. Q.W. Chen, D.W. Bahnemann, J. Am. Chem. Soc. 122, 970 (2000)

25. C. Huber, G. Wächtershäuser, Science 276, 245 (1997)

26. G.D. Cody, N.Z. Boctor, T.R. Filley, R.M. Hazen, J.H. Scott, A. Sharma, H.. Yoder Jr., Science 289, 1337 (2000)

27. C. Huber, G. Wächtershäuser, Science 314, 630 (2006)

28. J.L. Bada, B. Fegley Jr., S.L. Miller, A. Lazcano, H.J. Cleaves, R.M. Hazen, J. Chalmers, Science 315, 937 (2007)

29. Y. Furukawa, T. Sekine, M. Oba, T. Kakegawa, H. Nakazawa, Nature Geoscience 2, 62 (2009)

30. A. I. Oparin, Proikhozndenie Zhizni. Izd., (Moskowski Rabochi, 1924)

31. S.L. Miller, Science 117, 528 (1953)

32. S. Chang, in The chemistry of life's origin, pp. 259-300 (J.M. Greenberg, C.X. Mendoza-Gomez, V. Pirronello, editors, Kluwer Academic Publ., Dordrecht, 1993)

33. G. Schlesinger, S.L. Miller, J. Molec. Evol. 19, 376(1983)

34. H.J. Cleaves, J.H. Chalmers, A. Lazcano, S.L. Miller, J..L Bada, Orig. Life Evol. Biosph. 38, 105 (2008)

35. A.P. Johnson, H.J. Cleaves, J.P. Dworkin, D.P. Glavin, A. Lazcano, J.L. Bada, Science 322, $404(2008)$

36. F. Tian, O.B. Toon, A.A. Pavlov, A. De Sterck, Science 308, 1014 (2005)

37. B. Fegley Jr., R.G. Prinn, H. Hartman, G.H. Watkins, Nature 319, 305 (1986) 
38. A. Bar-Nun, N. Bar-Nun, S.H. Bauer, C. Sagan, Science 168, 470 (1970)

39. C.P. McKay, W.J. Borucki, Science 276, 390 (1997)

40. G. Ryder, Astrobiology 3, 3 (2003)

41. S. Pizzarello, Chem. Biodiv. 4, 680 (2007)

42. S. Pizzarello, E. Shock, Cold Spring Harb Perspect Biol 2:a002105 (2010)

43. H. Yabuta, L.B. William, G.D. Cody, C.M.O’D. Alexander, S. Pizzarello, Meteor Planet Sci 42, 37 (2007)

44. P.G. Stoks, A.W. Schwartz, Geochim. Cosmochim. Acta 46, 309 (1982)

45. D.W. Deamer, in The molecular origins of life: assembling pieces of the puzzle, pp. 189-205 (A. Brack, editor, Cambridge University Press, Cambridge, 1998)

46. P. Schmitt-Kopplin, Z. Gabelica, R.D. Gougeon, A. Fekete, B. Kanawati, M. Harir, I. Gebefuegi, G. Eckel, N. Hertkorn, Natl. Acad. Sci. USA 107, 2763 (2010)

47. J.R. Cronin, S. Pizzarello, Science, 275, 951 (1997)

48. S. Pizzarello, M. Zolensky, K.A. Turk, Geochim Cosmochim Acta 67, 1589 (2003)

49. S. Pizzarello, Y. Huang, Geochim Cosmochim Acta 69, 599 (2005)

50. T. Kawasaki, K. Hatase, Y. Fujii, K. Jo, K. Soai, S. Pizzarello, Geochim Cosmochim Acta 70, 5395 (2006)

51. W.A. Bonner, N.E. Blair, F.M. Dirbas, Origins Life 11, 119 (1981)

52. J. Bailey, A. Chrysostomou, J.H. Hough, T.M. Gledhill, A. McCall, S. Clark, F. Ménard, M. Tamura, Science 281, 672 (1998)

53. J. Bailey, Origins Life Evol. Biosphere 31, 167 (2001)

54. M. Bertrand, S. van der Gaast, F. Vilas, F. Hörz, G. Haynes, A. Chabin, A. Brack, F. Westall, Astrobiology 9, 943 (2009)

55. M. Maurette, Origins Life Evol. Biosphere 28, 385 (1998)

56. M. Maurette, A. Brack, Meteoritics \& Planet. Science 41, 5247 (2006)

57. K.L.F. Brinton, C. Engrand, D.P. Glavin, J.L. Bada, M. Maurette, Origins Life Evol. Biosphere 28, 413 (1998)

58. G. Matrajt, S. Pizzarello, S. Taylor, D. Brownlee, Meteoritics \& Planet. Science 39, 1849 (2004)

59. H.U. Keller, et al., Science, 327, 190 (2010)

60. I.N. Belskaya, et al., Astronomy \& Astrophysics 515, A29 (2010)

61. M.A. Barucci., et al., Astronomy \& Astrophysics 477, 665 (2008)

62. E. Bowell, et al., Icarus 35, 311 (1978)

63. G. Horneck, D. Stöffler, U. Eschweiler, U. Hornemann, Icarus, 149, 285 (2001)

64. C.-A.H. Roten, A. Gallusser, G.D. Borruat, S.D. Udry, D. Karamata, Bull. Soc. Vaud. Sci. nat. 86 (1), 1 (1998)

65. G. Horneck, Adv. Space Res. 23, 381 (1999)

66. R.L. Mancinelli, M.R. White, L.J. Rothschild, Adv. Space Res. 22, (3), 327 (1998)

67. P. Weber, J.M. Greenberg, Nature 316, 403 (1985)

68. C. Mileikowsky, F. Cucinotta, J.W. Wilson, B. Gladman, G. Horneck, L. Lindegren, J. Melosh, H. Rickman, M. Valtonen, J.Q. Zheng, Icarus 145, 391 (2000)

69. C.S. Cockell, A. Brack, D.D. Wynn-Williams, P. Baglioni, F. Brandstätter, R Demets, H.G.M., Edwards, A.L. Gronstal, G. Kurat, P. Lee, G.R. Osinski, D.A. Pearce, J.M. Pillinger, C.-A. Roten, S. Sancisi-Frey, Astrobiology 7, 1 (2007)

70. B.M. Jakosky, F. Westall, A. Brack, Mars, in Planets and Life, pp. 357-387 (W. T. Sullivan III, J. A. Baross, editors, Cambridge University Press, Cambridge, 2007)

71. W.V. Boynton et al., Science 297, 81 (2002)

72. J.P. Bibring et al., Science 312, 400 (2006)

73. http://www2.jpl.nasa.gov/snc/

74. A. Brack, C. Pillinger, Extremophiles 2, 313 (1998)

75. D.S. McKay, E.K. Gibson, K.L. Thomas-Keprta, H. Vali, C.S. Romanek, S.J. Clemett, X.D.F. Chellier, C.R. Maechling, R.N. Zare, Science 273, 924 (1996)

76. J.P. Bradley, R.P. Harvey, H.Y. McSween, Nature 390, 454 (1997)

77. F. Westall, P. Gobbi, G. Mazzotti, D. Gerneke, R. Stark, T. Dobrek, W. Heckl, SPIE, Instruments, Methods and Missions for Astrobiology, 3114, 225 (1998) 
78. A. Brack, P. Baglioni, G. Borruat, F. Brandstätter, R. Demets, H.G.M. Edwards, M. Genge, G. Kurat, M.F. Miller, E.M. Newton, C.T. Pillinger, C.-A. Roten, E. Wäsch, Planet. Space Science 50, 763 (2002)

79. F. Westall, A. Brack, B. Hofmann, G. Horneck, G. Kurat, J. Maxwell, G.G. Ori, C. Pillinger, F. Raulin, N. Thomas, B. Fitton, P. Clancy, D. Prieur, D. Vassaux, Planet. Space Sci. 48, 181 (2000)

80. V. Formisano, S. Atreya, T. Encrenaz, N. Ignatiev, M. Giuranna, Science 306, 1758 (2004)

81. M.J. Mumma, et al., Science 323, 1041 (2009)

82. J.F. Bell III, et al., J. Geophys. Res. 105 (E1), 1721 (2000)

83. G. Komatsu, G.G. Ori, Planet. Space Sci. 48, 1043 (2000)

84. L.J. Rothschild, R.L. Mancinelli, Nature 409, 1092 (2001)

85. C.S. Cockell, D.C. Catling, W. L. Davis, K. Snook, R. L. Kepner, P. Lee, C. P. McKay, Planet. Space Sci. 48, 343 (2000)

86. P.C. Magnani, T. Ylikorpi, G. Cherubini, A. Olivieri, Planet. Space Sci. 52, 79 (2004)

87. G.G. Ori, L. Marinangeli, G. Komatsu, Planet. Space Sci.48, 1043 (2000)

88. A. Brack, in A World Without Gravity, pp. 137-158 (G. Seibert et al., editors, ESA Publications Division, SP-1251, 2001)

89. F. Westall, Science 308, 366 (2005)

90. C. Sotin, D. Prieur, in Complete Course in Astrobiology, pp. 253-271 (G. Horneck, P. Rettberg, editors, Wiley-VCH, Weinheim, 2007)

91. C.F. Chyba, C. B. Phillips, in Planets and Life, pp. 388-423 (W.T. Sullivan III, J.A. Baross, editors, Cambridge University Press, Cambridge, 2007)

92. R.N. Clark, et al., Nature 435, (2005)

93. A. Coustenis, Astronomy \& Geophysics 48, 2.14 (2007)

94. J.I. Lunine, S.K. Atreya, Nature Geoscience 1, 159 (2008)

95. E.R. Stofan, et al., Icarus 185, 443 (2006)

96. E.R. Stofan, Nature 445, 61 (2007)

97. L.C. Wye, H.A. Zebker, R.D. Lorenz, Geophysical Research Letters, 36, L16201 (2009)

98. S. Wall, et al. Geophysical Research Letters 37, L05202 (2010)

99. F. Sohl, Science 327, 1367 (2010)

100. C.J. Hansen, L.W. Esposito, A.I.F. Stewart, B. Meinke, B. Wallis, J.E. Colwell, A.R. Hendrix, K. Larsen, W. Pryor, F. Tian, Nature 456, 477 (2008)

101. F. Spahn, K.-U Thiessenhusen, J.E. Colwell, R. Srama, E. Grun, Journal of Geophysical Research/Planets 104, 24111 (1999)

102. M. Bouhram, J.-J. Berthelier, J.-M. Illiano, H.T. Smith, E.C. Sittler, F.J. Crary, D.T. Young, Académie des Sciences. Comptes Rendus, Physique 6, (10) 1176 (2005)

103. J.-P. Beaulieu, A. Lecavelier des Etangs, C. Terquem, Extrasolar planets: Today and tomorrow (Astronomica Society of the Pacific Conference Series, 321, San Francisco, 2004)

104. P. Butler, in Planets and Life, pp. 444-458 (W.T. Sullivan III, J.A. Baross, editors, Cambridge University Press, Cambridge, 2007)

105. http://www.obspm.fr/encycl/f-encycl.html

106. F. Selsis, A. Léger, M. Ollivier, in Lectures in Astrobiology, pp. 385-423 (M. Gargaud, B. Barbier, H. Martin, J. Reisse, editors, Springer-Verlag Heidelberg, 2005)

107. J.C. Tarter, in Planets and Life, pp. 513-536 (W.T. Sullivan III, J.A. Baross, editors, Cambridge University Press, Cambridge, 2007) 